Observer-based consensus cont rol st $r$ at egy for mul $\mathrm{t} i$-agent syst em wi th communi cat $i$ on ti me del ay

\begin{tabular}{|c|c|}
\hline 著者 & Yoshi oka Chi ka, Namer i kawa Tor u \\
\hline $\begin{array}{l}\text { jour nal or } \\
\text { publ i cat } i \text { on } t i t l e\end{array}$ & $\begin{array}{l}\text { Proceedi ngs of the I EEE I nt er nat i onal } \\
\text { Conf er ence on Cont rol Appl i cat i ons }\end{array}$ \\
\hline page $r$ ange & $1037-1042$ \\
\hline year & 2008-01- 01 \\
\hline URL & ht t p: //hdl . handl e. net /2297/12039 \\
\hline
\end{tabular}




\title{
Virtual Structure Based Target-enclosing Strategies for Nonholonomic Agents
}

\author{
Hiroki Kawakami and Toru Namerikawa
}

\begin{abstract}
In this paper, we discuss a target-enclosing problem for a group of multiple nonholonomic agents in a plane. The proposed strategies guarantee that multiple agents' coordination finally results in a circular formation enclosing the targetobject which moves in the plane. Firstly, virtual agents for the feedback linearization of the real nonholonomic agents are introduced. Secondly, we propose the target-enclosing control laws based on the consensus algorithm to the virtual agents. Algebraic graph theory and consensus algorithm are employed to prove convergence and stability of the enclosing problem. Finally, experiments are provided to demonstrate the effectiveness of the proposed control laws.
\end{abstract}

\section{INTRODUCTION}

In recent years, there have been increasing research interests in the distributed cooperative control of multi-agent systems [1]-[4]. Several research groups developed the coordination control strategies that achieve a capturing formation around a target object (specific area) by multiple mobile agents using local information [5]-[11]. Owing to the broad range of applications (e.g. investigations in hazardous environments, mobile sensor networks and security systems), the task of capturing target-object is researched in the distributed cooperative control of multi-agent systems.

The capturing the target-object is divided into two problems, grasping behavior and enclosing behavior. The grasping behavior is the object-closure condition in decentralized form in [5]. On the other hand, the enclosing behavior is that multiple agents are controlled in a distributed manner to converge to an assigned formation while tracking the moving target object. Kobayashi et al. [7] proposed the decentralized grasping control law using the concept of force-closure and enclosing control law based on a gradient decent method for multiple agents with local information in $2 \mathcal{D}$ plane. In their method, each agent requires local information about target object and two neighbor agents.

Marshall et al. [12][13] proposed a cyclic pursuit based formation control strategies for multiple mobile agents moving in a plane. They showed that the multiple agents finally can assemble in a circular formation that is similar to that of [7]. In [6], Kim et al. proposed a distributed cooperative control method based on a cyclic pursuit strategy in a targetenclosing task in $3 \mathcal{D}$ space by multi-agent systems. In the above method, each agent's behavior is decided using the local information about target-object and one neighbor agent.

H. Kawakami and T. Namerikawa are with Division of Electrical Engineering and Computer Science, Graduate School of Natural Science and Technology, Kanazawa University, Kakuma-machi, Kanazawa 9201192 JAPAN. hirokiescl.ec.t.kanazawa-u.ac.jp and toru@t.kanazawa-u.ac.jp
In [6][7], however, enclosing strategies for multiple agents with nonholonomic constraints (e.g. two-wheeled vehicles and AUVs) have not been considered and in their method, all agents require the states of the target object. In addition, the information exchange topologies between the agents are limited to the cycle graphs. i.e. enclosing the target-object cannot be achieved with the information exchange topologies except cycle graphs.

On the other hand, consensus algorithm based formation control strategies for multi-agent systems are proposed in [14]-[17]. Ren [15] proposed the formation control strategies for multi-agent systems where the information states of each agent approach a common time-varying reference state. Namerikawa et al. [16] proposed virtual structure based formation control strategies for nonholonomic multi-vehicle systems.

Most consensus algorithm results related to cooperative control are obtained for linear agents. However, most practical cooperative control applications involve systems that are nonlinear and nonholonomic. Therefore, it is necessary to discuss cooperative control of nonholonomic agents. There have been some previous research works [10], [18], [19] which treated cooperative control of multiple nonholonomic agents. In this paper, we propose virtual structure based target-enclosing strategies for multiple nonholonomic agents which are controlled to converge to the formation while they are tracking the target-object moving in $2 \mathcal{D}$ plane. Firstly, we introduce a virtual agent for the feedback linearization of the real agent. Secondly, we propose the target-enclosing control laws based on the consensus algorithm to the virtual agents. Finally, experiments are provided to demonstrate the effectiveness of the proposed control laws.

This paper is organized as follows. Section II introduces background and preliminaries (algebraic graph theory and consensus algorithm). Section III introduces the virtual structures corresponding to real agents and real target-object respectively and control objectives of the enclosing behavior. Section IV describes the proposed enclosing strategies for multiple nonholonomic agents. In Section V, we validate our results by experiments. Finally, we summarize the obtained results and future works in Section VI.

\section{PRELIMINARIES}

Let $\mathscr{G}=(\mathscr{V}, \mathscr{E})$ denoted a graph with the set of vertices $\mathscr{V}=\{1,2, \cdots, n\}$ and the set of edges $\mathscr{E} \subseteq \mathscr{V} \times \mathscr{V}$ [20]. The graph is divided into undirected graphs and directed graphs (digraphs). It is natural to model information exchange between agents by graphs. If any two vertices $i, j \in \mathscr{V}$ and 
$(i, j) \in \mathscr{E}$, the vertex $j$ is neighbors of vertex $i$ is denoted by $i \sim j$. The set of neighbors of vertex $i$ is denoted by

$$
\mathcal{N}_{i}=\{j \mid i \sim j\} \subseteq\{1, \ldots, n\} \backslash\{i\}
$$

An undirected graph is called connected if there is an edge between any distinct pair of vertices. An directed graph is called strongly connected if there is a directed edge from every from every other vertices, while a directed graph is called balanced if $\sum_{j \neq i} a_{i j}=\sum_{j \neq i} a_{j i}, i \in \mathscr{V}$. A directed tree is a directed graph, where every vertex has exactly one parent except for one vertex, called root, which has no parent, and the root has a directed path to every other vertex [15]. Note that in a directed tree, each edge has a natural orientation away from the root, and no cycle exists. In the case of undirected graphs, a tree is a graph in which every pair of vertices is connected by exactly one path. A directed spanning tree of a directed tree formed by graph edges that connect all of the vertices of the graph. Note that the condition that a digraph has a directed spanning tree is equivalent to the case that there exists as least a vertex having a directed path to all of the other vertices. In the case of undirected graphs, having an undirected spanning tree is equivalent to being connected. The adjacency matrix $\mathcal{A}(\mathscr{G})=\left[a_{i j}\right] \in \mathbb{R}^{n \times n}$ is defined as $a_{i i}=0$ and $a_{i j}=1$ if $(j, i) \in \mathscr{E}$ where $i \neq j$. The adjacency matrix of a undirected graph is defined accordingly except that $a_{i j}=a_{j i},{ }_{i} i \neq j$, since $(j, i) \in \mathscr{E}$ implies $(i, j) \in \mathscr{E}$. The degree of vertex $i$ is the number of its neighbors $\left|\mathcal{N}_{i}\right|$ and is denoted by $\operatorname{deg}(i)$. The degree matrix of graph $\mathscr{G}$ is diagonal matrix defined as $\mathcal{D}(\mathscr{G})=\left[d_{i j}\right] \in \mathbb{R}^{n \times n}$ where

$$
d_{i j}=\left\{\begin{array}{lll}
\operatorname{deg}(i) & , & i=j \\
0, & i \neq j
\end{array}\right.
$$

Laplacian matrix of the graph $\mathscr{G}$ is defined by

$$
\mathcal{L}(\mathscr{G})=\mathcal{D}(\mathscr{G})-\mathcal{A}(\mathscr{G})=\left[l_{i j}\right] \in \mathbb{R}^{n \times n}
$$

For an undirected graph, the Laplacian matrix $\mathcal{L}$ is symmetric positive semi-definite. This property does not hold for a digraph Laplacian matrix. An important feature of $\mathcal{L}$ is that all the row sums of $\mathcal{L}$ are zero and thus $\mathbf{1}_{n}=$ $\left[\begin{array}{llll}1 & 1 & \cdots & 1\end{array}\right]^{T} \in \mathbb{R}^{n}$ is eigenvector of $\mathcal{L}$ associated with the eigenvalue $\lambda(\mathcal{L})=0$.

\section{Problem Formulation}

\section{A. Virtual Structures}

In this subsection, we consider $n$ nonholonomic mobile agents (see the lower left at Figure 1(a)). $i^{\text {th }}$ nonholonomic mobile agent is modeled by the following nonlinear ordinary differential equations

$$
\left[\begin{array}{c}
\dot{x}_{i} \\
\dot{y}_{i} \\
\dot{\theta}_{i}
\end{array}\right]=\left[\begin{array}{cc}
\cos \theta_{i} & 0 \\
\sin \theta_{i} & 0 \\
0 & 1
\end{array}\right]\left[\begin{array}{c}
v_{i} \\
\omega_{i}
\end{array}\right]
$$

where $r_{i}=\left[x_{i} y_{i}\right]^{T} \in \mathbb{R}^{2}$ is the position of $i^{\text {th }}$ agent, $\theta_{i} \in$ $[0,2 \pi)$ is the orientation, $v_{i} \in \mathbb{R}$ is the forward velocity and

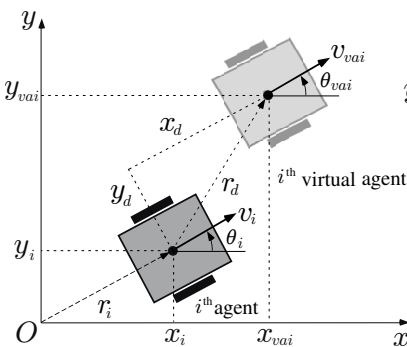

(a)

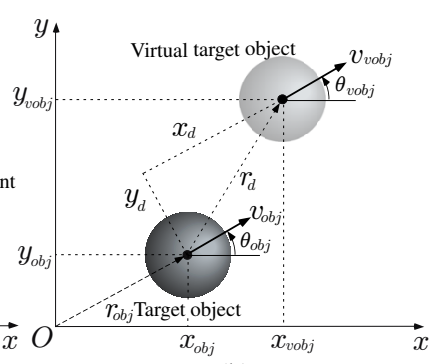

(b)
Fig. 1. Definition of virtual structures. (a) Real agent and virtual agent, (b) Real target-object and virtual target-object.

$\omega_{i} \in \mathbb{R}$ is the angular velocity. The agents has the following nonholonomic constraint of pure rolling and non-slipping.

$$
\dot{x}_{i} \sin \theta_{i}-\dot{y}_{i} \cos \theta_{i}=0
$$

We define the virtual agent (see the upper right at Figure 1(a)) corresponding to the real agent (4). Then, the relation between $i^{\text {th }}$ real agent and $i^{\text {th }}$ virtual agent is given by

$$
\left[\begin{array}{c}
x_{v a i} \\
y_{v a i} \\
\theta_{v a i}
\end{array}\right]=\left[\begin{array}{c}
x_{i}+x_{d} \cos \theta_{i}-y_{d} \sin \theta_{i} \\
y_{i}+x_{d} \sin \theta_{i}+y_{d} \cos \theta_{i} \\
\theta_{i}
\end{array}\right],
$$

where $r_{v a i}=\left[\begin{array}{ll}x_{v a i} & y_{v a i}\end{array}\right]^{T} \in \mathbb{R}^{2}$ and $\theta_{v a i} \in[0,2 \pi)$ are, respectively, the position, the orientation of $i^{\text {th }}$ virtual agent. $r_{d}=\left[\begin{array}{ll}x_{d} & y_{d}\end{array}\right]^{T} \in \mathbb{R}^{2}$ is the distance between the real agent and the virtual agent. Then, the derivative of eq. (6) is given as

$$
\begin{aligned}
{\left[\begin{array}{c}
\dot{x}_{v a i} \\
\dot{y}_{v a i} \\
\dot{\theta}_{v a i}
\end{array}\right] } & =\left[\frac{B_{i}}{B_{\theta}}\right]\left[\begin{array}{c}
v_{i} \\
\omega_{i}
\end{array}\right] \\
& =B\left(\theta_{i}\right) u_{i}
\end{aligned}
$$

where $u_{i}=\left[v_{i} \omega_{i}\right]^{T} \in \mathbb{R}^{2}$ is the control input to $i^{\text {th }}$ agent and

$$
\begin{aligned}
B_{i} & =\left[\begin{array}{cc}
\cos \theta_{i} & -x_{d i} \sin \theta_{i}-y_{d i} \cos \theta_{i} \\
\sin \theta_{i} & x_{d i} \cos \theta_{i}-y_{d i} \sin \theta_{i}
\end{array}\right] \\
B_{\theta} & =\left[\begin{array}{ll}
0 & 1
\end{array}\right]
\end{aligned}
$$

If we assume $x_{d i} \neq 0, B_{i}$ is a regular matrix.

Similarly, we consider the virtual target-object corresponding to real target-object (see Figure 1(b)). Then, the states of virtual target-object are given as well as the case of virtual agents as follows

$$
\left[\begin{array}{l}
x_{v o b j} \\
y_{v o b j} \\
\theta_{v o b j}
\end{array}\right]=\left[\begin{array}{c}
x_{o b j}+x_{d} \cos \theta_{o b j}-y_{d} \sin \theta_{o b j} \\
y_{o b j}+y_{d} \sin \theta_{o b j}+y_{d} \cos \theta_{o b j} \\
\theta_{o b j}
\end{array}\right],
$$

where $r_{o b j}=\left[\begin{array}{ll}x_{o b j} & y_{o b j}\end{array}\right]^{T} \in \mathbb{R}^{2}$ is the position of real targetobject, $r_{v o b j}=\left[\begin{array}{ll}x_{v o b j} & y_{v o b j}\end{array}\right]^{T} \in \mathbb{R}^{2}$ is the position of virtual target-object, $\theta_{o b j} \in[0,2 \pi)$ is the moving orientation of real target-object and $\theta_{v o b j} \in[0,2 \pi)$ is the moving orientation of virtual target-object. 


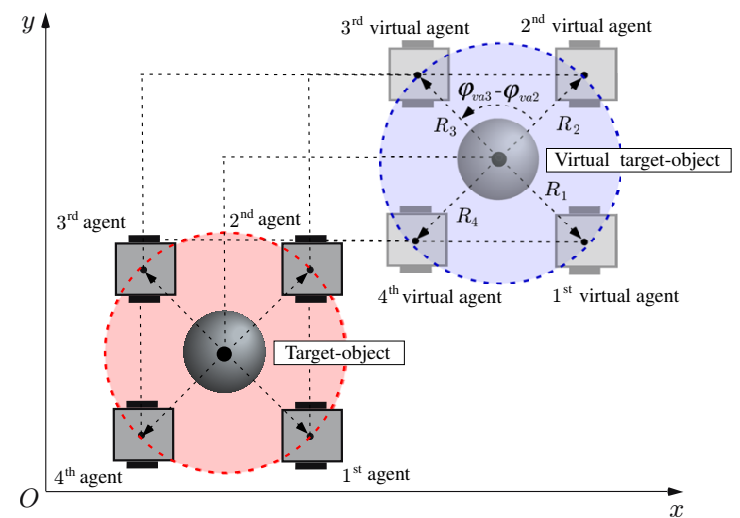

Fig. 2. An example of $n=4$ virtual agents enclosing virtual target object.

\section{B. Control Objectives}

We first define the position in which $i^{\text {th }}$ agent encloses the target-object as enclosing position $R_{i} \in \mathbb{R}^{2}$. This paper only considers the equal convergence positions for all agents; i.e.,

$$
\left\|R_{1}\right\|=\left\|R_{2}\right\|=\cdots=\left\|R_{n}\right\|=\xi=\text { const. }
$$

where $\|\cdot\|$ is the Euclidean norm, $\xi \in \mathbb{R}$ is the enclosing radius. Let $\phi_{\text {vai }} \in \mathbb{R}$ denote the counterclockwise angle of $i^{\text {th }}$ virtual agent around the center of the virtual target-object. Control objectives for virtual structure based enclosing behavior can be formulated as follows (see Figure 2) ;

$$
\begin{aligned}
& \text { A1) }\left\|r_{v a i}-r_{v o b j}\right\| \rightarrow \xi[\mathrm{m}] \quad \text { as } \quad t \rightarrow \infty, \\
& \text { A2) }\left\|\phi_{v a(i+1)}-\phi_{v a i}\right\| \rightarrow \frac{2 \pi}{n}[\mathrm{rad}] \quad \text { as } t \rightarrow \infty, \\
& \text { A3) }\left\|\theta_{v a i}-\theta_{v o b j}\right\| \rightarrow 0[\mathrm{rad}] \quad \text { as } \quad t \rightarrow \infty, \\
& \quad \text { for } i=1, \cdots, n .
\end{aligned}
$$

In case of $i=n, n+1=1$.

\section{Virtual Structure Based Target Enclosing STRATEGIES}

\section{A. Enclosing Control Law for Balanced Relative Positions}

In this subsection, we propose the target-enclosing control law 1 for balanced relative positions. If the average of initial positions of all virtual agents is equal to the initial position of virtual target-object, the control law 1 achieves the above control objectives A1)-A3). Here, we make the following Assumption 1-2.

Assumption 1: Information exchange topology between agents is a connected graph or a balanced graph.

Assumption 2: The target-object moves at the forward speed $v_{o b j} \neq 0$ and all agents can acquire the target object's position $r_{o b j}$ and its derivative $\dot{r}_{o b j}$ from the target object.

Then, the proposed enclosing control law 1 for $i^{\text {th }}$ agent is described as

$$
u_{i}=B_{i}^{-1}\left[-k \sum_{j \in \mathcal{N}_{i}}\left\{\hat{r}_{v a i}-\hat{r}_{v a j}\right\}+\dot{r}_{v o b j}\right],
$$

where $u_{i} \in \mathbb{R}^{2}$ is an input, $k \in \mathbb{R}$ is a constant controller gain, $R_{i} \in \mathbb{R}^{2}$ is the enclosing position, $\mathcal{N}_{i}$ is the set of neighboring agents of $i^{\text {th }}$ agent, $B_{i}^{-1}$ is the inverse matrix of $B_{i}$ and $\hat{r}_{v a i} \doteq r_{v a i}-R_{i}$. This enclosing control law 1 requires relative distances between the agent and the other agent, the derivative of $r_{o b j}$ and the orientation $\theta_{i}$. Next, we require the following Lemma 1-2 from [3][16].

Lemma 1 : [16] Consider an information exchange topology $\mathscr{G}$ of $n$ agents with the Laplacian matrix $\mathcal{L}(\mathscr{G})$ applying the following consensus algorithm

$$
\dot{\zeta}=-\left(\mathcal{L} \otimes I_{m}\right) \zeta
$$

Suppose $\mathscr{G}$ is a connected graph or a strongly connected graph. Then, a consensus is asymptotically reached for initial states of all agents ;

$$
\zeta \rightarrow\left(\zeta_{r 1} \zeta_{l 1}^{T} \otimes I_{m}\right) \zeta(0)=\mathbf{1}_{n} \otimes \alpha \quad \text { as } t \rightarrow \infty
$$

Let $\zeta_{r 1}, \zeta_{l 1}$ be a right / left eigenvector of laplacian matrix $\mathcal{L}$ is associated with eigenvalue $0 . \zeta_{r 1}$ and $\zeta_{l 1}$ satisfying $\zeta_{l 1}^{T} \zeta_{r 1}=1$ and $\zeta_{l 1}^{T} \mathbf{1}_{n}=1$, where $\zeta_{i} \in \mathbb{R}^{m}, \zeta=$ $\left[\begin{array}{llll}\zeta_{1}^{T} & \zeta_{2}^{T} & \cdots & \zeta_{n}^{T}\end{array}\right]^{T} \in \mathbb{R}^{n m}, \otimes$ denotes the Kronecker product and $\alpha \in \mathbb{R}^{m}$ is the group decision vector.

Lemma 2 : [3] If an information exchange topology $\mathscr{G}$ satisfies Assumption 1, an average-consensus is asymptotically reached and

$$
\alpha=\frac{1}{n} \sum_{i=1}^{n}\left\{\zeta_{i}(0)\right\} .
$$

Now, we have the following Theorem 1.

Theorem 1 : Consider the system of $n$ virtual agents (7) and the virtual target-object (10). We apply the enclosing control law 1 (12) to the system. If the control law 1 satisfies $k>0$, Assumption 1-2 and equ. (16)

$$
\frac{1}{n} \sum_{i=1}^{n} \hat{r}_{v a i}(0)=r_{v o b j}(0),
$$

then the control law 1 asymptotically achieves the control objectives A1)- A3).

$$
\begin{aligned}
& \text { Proof: } \\
& \text { Let } r_{v a} \doteq\left[\begin{array}{llll}
r_{v a 1}^{T} & \cdots & r_{v a n}^{T}
\end{array}\right]^{T}, \hat{r}_{v a} \doteq \\
& {\left[\begin{array}{ccccc}
\hat{r}_{v a 1}^{T} & \cdots & \hat{r}_{v a n}^{T}
\end{array}\right]^{T}, r_{v a}^{\prime} \doteq\left[\begin{array}{llll}
r_{v a 1}^{\prime} & \cdots & r_{v a n}^{\prime}
\end{array}\right]^{T},} \\
& R \doteq\left[\begin{array}{llll}
R_{1}^{T} & \cdots & R_{n}^{T}
\end{array}\right]^{T} \text { and } u \doteq\left[\begin{array}{llll}
u_{1}^{T} & \cdots & u_{n}^{T}
\end{array}\right]^{T} \text {. Also }
\end{aligned}
$$
let

$$
\left\{\begin{array}{l}
r_{v a}^{\prime}=\hat{r}_{v a}-\mathbf{1}_{n} \otimes r_{v o b j}=r_{v a}-R-\mathbf{1}_{n} \otimes r_{v o b j} \\
\dot{r}_{v a}^{\prime}=\dot{\hat{r}}_{v a}-\mathbf{1}_{n} \otimes \dot{r}_{v o b j}=\dot{r}_{v a}-\dot{R}-\mathbf{1}_{n} \otimes \dot{r}_{v o b j}
\end{array}\right.
$$

Then, enclosing-control input $u$ of $n$ agents is described as

$$
u=\oplus \sum_{i=1}^{n} B_{i}^{-1}\left\{-k\left(\mathcal{L} \otimes I_{2}\right) r_{v a}^{\prime}+\mathbf{1}_{n} \otimes \dot{r}_{v o b j}\right\},
$$

where $u \in \mathbb{R}^{2 n}, \oplus \sum_{i} a_{i}$ has diagonal block elements $a_{i}$ and $\mathcal{L}$ is Laplacian matrix of information exchange topology of 
the agents. The virtual agent (7) can be written in $n$ virtual agents form as

$$
\dot{r}_{v a}=\oplus \sum_{i=1}^{n} B_{i} u .
$$

With (18), eq. (19) is given by

$$
\dot{r}_{v a}^{\prime}=-k\left(\mathcal{L} \otimes I_{2}\right) r_{v a}^{\prime} .
$$

From Lemma 1 , we know that $r_{v a}^{\prime} \rightarrow \mathbf{1} \otimes \alpha$ as $t \rightarrow \infty$, therefore

$$
r_{v a}-\mathbf{1} \otimes r_{v o b j} \rightarrow \mathbf{1} \otimes \alpha+R \quad \text { as } \quad t \rightarrow \infty,
$$

where $\alpha \in \mathbb{R}^{2}$ is a group decision vector. From Assumption 2 , if the information exchange topology is connected graph or balanced graph, the group decision $\alpha$ asymptotically reaches the average of initial positions of all agents. Thus, we have

$$
\alpha=\frac{1}{n} \sum_{i=1}^{n} r_{v a i}^{\prime}(0)=\frac{1}{n}\left\{\sum_{i=1}^{n} \hat{r}_{v a i}(0)-n \cdot r_{v o b j}(0)\right\} \text {. }
$$

If initial positions of all agents satisfy $\alpha=0$, eq. (21) can be rewritten as

$$
r_{v a i}-r_{v o b j} \rightarrow R_{i} \quad \text { as } \quad t \rightarrow \infty .
$$

In addition, if we design enclosing position $R_{i}$ as follows

$$
R_{i}=\xi\left[\cos \frac{2 \pi(i-1)}{n} \sin \frac{2 \pi(i-1)}{n}\right]^{T}, \dot{R}_{i}=0
$$

we obtain $\left\|r_{v a i}-r_{v o b j}\right\| \rightarrow \xi[\mathrm{m}]$ and $\left\|\phi_{v a(i+1)}-\phi_{v a i}\right\| \rightarrow$ $\frac{2 \pi}{n}[\mathrm{rad}]$ as $t \rightarrow \infty, i \in \mathscr{V}$ simultaneously.

Proposition 1 : The steady orientation of virtual targetobject and convergence positions of virtual agents are assumed as $\dot{\theta}_{v o b j}=0$ and $\hat{r}_{v a i}-R_{i}=\hat{r}_{v a j}-R_{j}$. Then, the orientations of all virtual agents achieve the orientation of virtual target-object. i.e. $\theta_{\text {vai }} \rightarrow \theta_{\text {vobj }}, i \in \mathscr{V}$.

Proof: From (7) and (12), the derivative of the orientation of $i^{\text {th }}$ virtual agent is given by

$$
\dot{\theta}_{v a i}=-\frac{\left\|\dot{r}_{v o b j}\right\|}{x_{d}} \sin \left(\theta_{v a i}-\theta_{v o b j}\right),
$$

which implies that $\theta_{v a i} \rightarrow \theta_{v o b j}$ as $t \rightarrow \infty, i \in \mathscr{V}$.

\section{B. Enclosing Control Law for Any Initial Position}

In this subsection, we propose the control law 2 which achieves the above control objectives A1)-A3) for any initial relative positions between all agents and the target-object. Then, the proposed enclosing control law 2 for $i^{\text {th }}$ agent is described as

$$
\begin{aligned}
u_{i}=B_{i}^{-1}\left[-k_{a}\left\{\hat{r}_{v a i}-r_{v o b j}\right\}\right. & \\
& \left.\quad-k_{b} \sum_{j \in \mathcal{N}_{i}}\left\{\hat{r}_{v a i}-\hat{r}_{v a j}\right\}+\dot{r}_{v o b j}\right],
\end{aligned}
$$

where $k_{a}, k_{b} \in \mathbb{R}$ are constroller gains. Now, we have the following Theorem 2.
Theorem 2 : Consider the system of $n$ virtual agents (7) and the virtual target-object (10). We apply the enclosing control law 2 (26) to the system. If the control law 2 satisfies $k_{a}, k_{b}>0$ and assumption 1-2, the control law 2 asymptotically achieves the control objectives A1)- A2).

Proof: With (26), eq. (19) can be written in matrix form as

$$
\dot{r}_{v a}^{\prime}=-k_{a} r_{v a}^{\prime}-k_{b}\left(\mathcal{L} \otimes I_{2}\right) r_{v a}^{\prime}=-\mathcal{P} r_{v a}^{\prime}
$$

where $\mathcal{P} \in \mathbb{R}^{2 n}$. We know that

$$
r_{v a}-\mathbf{1} \otimes r_{v o b j} \rightarrow R \quad \text { as } \quad t \rightarrow \infty
$$

Therefore, if we design the following enclosing position $R_{i}$ (24), the enclosing control law 2 asymptotically achieves the control objectives A1)-A2) for any initial relative positions between all agents and the target-object.

\section{Enclosing Control Law for Leader-follower Systems}

In this subsection, we discuss the case that a portion of agents has access to the target-object (i.e. the leader-follower systems). It is generally difficult to acquire information about the target-object moving in actual environment. Here, we make the following Assumptions 3-4.

Assumption 3 : Information exchange topology between agents has a spanning tree.

Assumption 4 : The target-object moves at the forward speed $v_{o b j} \neq 0$, the leaders can acquire the position $r_{o b j}$ of target object and its derivative $\dot{r}_{o b j}$. $i^{\text {th }}$ follower can acquire the position $r_{v a j}$ of the adjacent $j^{\text {th }}$ follower and its derivative $\dot{r}_{v a j}$.

We propose the target-enclosing control law 3 based on [15]. The proposed control law for the leader-agents and followeragents is described as

- Control input for the $i^{\text {th }}$ leader-agent

$$
\begin{aligned}
u_{i}=\frac{B_{i}^{-1}}{1+\sum_{j \in \mathcal{N}_{i}} a_{i j}}\left[-k_{i}\left(\hat{r}_{v a i}-r_{v o b j}\right)+\dot{r}_{v o b j}\right. \\
\left.-\sum_{j \in \mathcal{N}_{i}}\left\{k_{i}\left(\hat{r}_{v a i}-\hat{r}_{v a j}\right)+\dot{r}_{v a j}\right\}\right]
\end{aligned}
$$

- Control input for the $i^{\text {th }}$ follower-agent

$$
u_{i}=\frac{B_{i}^{-1}}{\sum_{j \in \mathcal{N}_{i}} a_{i j}}\left[\sum_{j \in \mathcal{N}_{i}}-k_{i}\left(\hat{r}_{v a i}-\hat{r}_{v a j}\right)+\dot{r}_{v a j}\right]
$$

where $k_{i} \in \mathbb{R}$ are controller gain. Here, we have the following Theorem 3.

Theorem 3 : Consider the system of $n$ virtual agents $(p \geq 1$ leaders and $q(=n-p)$ followers $)$ and the virtual targetobject (10). We apply the enclosing control law 3 (29)(30) to the system. If the control law 3 (29)(30) satisfies $k_{i}>0$, Assumption 3-4, the control law 3 asymptotically achieves the control objectives A1)- A2). 


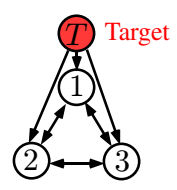

(a)

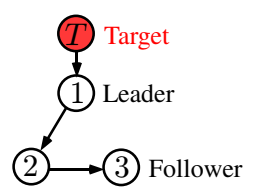

(b)
Fig. 3. Information exchange topologies

TABLE I

CONTROLlER PARAMETERS FOR EXPERIMENTS

\begin{tabular}{c||c}
\hline Case $1: k_{a}, k_{b}$ & 0.3 \\
\hline Case $2: k_{i}$ & 0.3 \\
\hline$r_{d}[\mathrm{~m}]$ & {$[0.2,0]$} \\
\hline$R_{1}[\mathrm{~m}]$ & {$[1 / 3,0]$} \\
\hline$R_{2}[\mathrm{~m}]$ & {$[1 / 6, \sqrt{3} / 6]$} \\
\hline$R_{3}[\mathrm{~m}]$ & {$[-1 / 6, \sqrt{3} / 6]$} \\
\hline
\end{tabular}

Proof: For (29)(30), let $r_{v o b j} \doteq \hat{r}_{v a(n+1)}$. We rewrite equ. (29)(30) as

$$
\sum_{j=1}^{n+1} a_{i j}\left(\dot{\hat{r}}_{v a i}-\dot{\hat{r}}_{v a j}\right)=-k_{i} \sum_{j=1}^{n+1} a_{i j}\left(\hat{r}_{v a i}-\hat{r}_{v a j}\right) .
$$

Eq. (31) can be written in matrix form as $\left(\mathcal{L}_{n+1} \otimes I_{2}\right) \dot{\hat{r}}_{\text {vai }}=-k_{i}\left(\mathcal{L}_{n+1} \otimes I_{2}\right) \hat{r}_{\text {vai }}$, which implies that $\left(\mathcal{L}_{n+1} \otimes I_{2}\right) \hat{r}_{v a i} \rightarrow 0$ and $\hat{r}_{v a i} \rightarrow r_{v o b j}$ as $t \rightarrow \infty$, since $\hat{r}_{v a(n+1)} \doteq r_{v o b j}$. Therefore, if we design the following enclosing position $R_{i}$, the enclosing control law 3 asymptotically achieves the control objectives A1) - A2).

Note that the control law 2 is the case that all agents can acquire the target-object.

\section{Evaluation By CONTROL EXPERIMENTS}

Figure 3 shows the two kinds of the information exchange topologies.

In experiments (see Figure 4), the four two-wheeled vehicles (nonholonomic agents) and the same one vehicle for the target object are used. The vehicles used in the experiments are controlled by a digital signal processor (DSP) from dSPACE Inc., which utilizes a PowerPC running at 3.2 $[\mathrm{GHz}]$. Control programs are written in MATLAB/Simulink, and implemented on the DSP using the Real-Time Workshop and dSPACE software which includes ControlDesk, RealTime Interface. A CCD camera is mounted above the vehicles. The video signals are acquired by a frame grabber board PicPort and image processing software HALCON. The sampling time of the controller is 0.2 [s]. The position, velocity and orientation of the vehicles are calculated by using the image processing. In the experiments, it is assumed that enclosing was achieved when it satisfies the following conditions, because a complete enclosing behavior cannot be achieved by restricting the experimental environment.

- $\left\|r_{i}^{\prime}\right\| \leq 0.06[\mathrm{~m}]$ ( within $20[\%]$ )

- $\phi_{(i+1)}-\phi_{i} \leq 2 \pi / 3 \pm 0.3[\mathrm{rad}]$ ( within 15 [\%] )

- $\left\|\theta_{i}-\theta_{o b j}\right\| \leq 0.3[\mathrm{rad}]$

Then, enclosing performances of case 1 and case 2 are compared on the above condition.

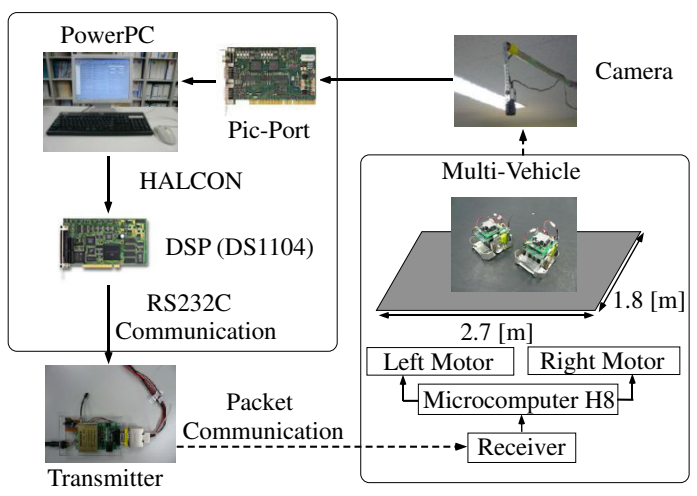

Fig. 4. Experimental setup

Case 1: To illustrate the enclosing performances of the proposed control law 2, the experiments are carried out in which $n=3$ agents and one target-object with information exchange topology in Figure 4 (a). The controller parameters of the experiments are given as shown in Table I. The velocity of the target-object is $0.04[\mathrm{~m} / \mathrm{s}]$. The experiment results are shown in Figures 5-6. Figure 5 illustrates the trajectories of the three agents and the target object. Figure 6 illustrates $r_{i}-r_{o b j}, \phi_{(i+1)}-\phi_{i}$ and $\theta_{i}-\theta_{o b j}$ of each agent. They show that all agents converge to a circular formation around the target object at about 18 [s]. The above experimental results demonstrate that the control objectives A1)-A3) are achieved.

Case 2: To illustrate the enclosing performances of the proposed control law 3, an experiments are carried out in which $n=3$ agents (one leader and two followers) and a target-object with information exchange topology in Figure 4 (b). The controller parameters of the experiments are given as shown in Table I. The velocity of the target-object is $0.04[\mathrm{~m} / \mathrm{s}]$. The experiment results are shown in Figures 7-8. Figure 7 illustrates the trajectories of the three agents and the target object. Figure 8 illustrates $r_{i}-r_{o b j}, \phi_{(i+1)}-\phi_{i}$ and $\theta_{i}-\theta_{o b j}$ of each agent. The above experimental results demonstrate that the control objectives A1)-A3) are achieved. They show that all agents converge to a circular formation around the target object at about 26 [s]. The convergence speeds for Case 2 (26 [s]) are slow compared with Case 1 (18 [s]), because in Case 1 and Case 2, the communication costs between the agents are different.

VI. CONCLUSions AND Future WORKS

In this paper, we have proposed virtual structure based target-enclosing strategies for multiple nonholonomic agents. Firstly, virtual agents for the feedback linearization of the real nonholonomic agents were introduced. Secondly, we proposed the target-enclosing control laws based on the consensus algorithm to the virtual agents. Algebraic graph theory and consensus algorithm were employed to prove convergence and stability of the enclosing problem. Finally, experiments are provided to demonstrate the effectiveness of the proposed control laws. Future work will address target enclosing for the target-object which is at a stand still. In addition, the extension of the current work to target enclosing 


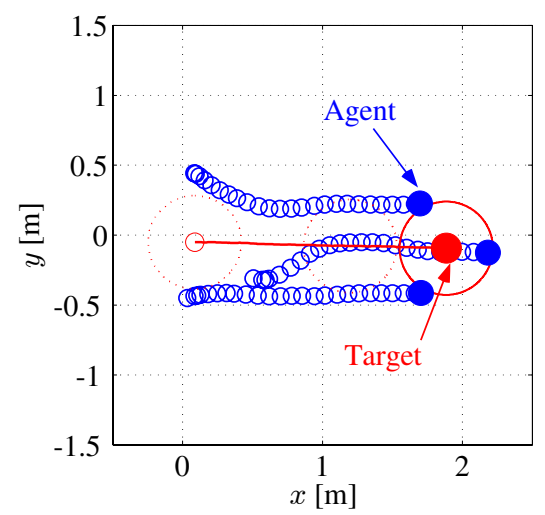

Fig. 5. Case 1 : Trajectories of agents and target-object

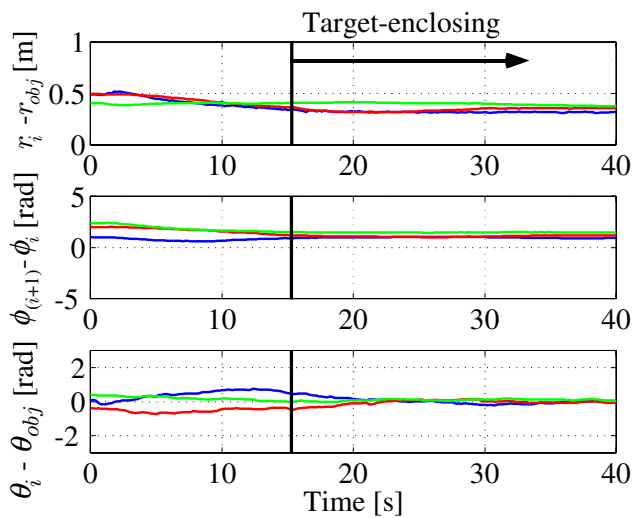

Fig. 6. Case 1 : Time plots of $r_{i}-r_{o b j}, \phi_{(i+1)}-\phi_{i}$ and $\theta_{i}-\theta_{o b j}$

under switching information exchange topologies will also be a topic of future work.

\section{REFERENCES}

[1] R. O. Saber, J. A. Fax and R. M. Murray : Consensus and Cooperation in Networked Multi-Agent Systems, in Proc. of the IEEE, Vol. 95, No. 1, pp. 215-233, 2007.

[2] V. Kumar, N. Leonard and A. S. Morse, eds. : Cooperative Control : A Post-workshop Volume 2003 Block Island Workshop On Cooperative Control, Lecture Notes in Control and Information Sciences, Vol. 309, Springer, 2004.

[3] R. O. Saber and R. M. Murray : Consensus Problems in Networks of Agents With Switching Topology and Time-Delays, IEEE Trans. Automatic Control, Vol. 49, no. 9, pp. 1520-1533, 2004.

[4] H. Tanner, A. Jadbabaie, and G. J. Pappas : Flocking in fixed and switching networks, IEEE Trans on Automatic Control, Vol. 52, No. 5, pp. 863-868, 2007.

[5] G. A. S. Pereira, V. Kumar and M. F. M. Campos : Decentralized Algorithms for Multi-Robot Manipulation via Caging. The International Journal of Robotics Research (IJRR), 23-7, 2004.

[6] T. H. Kim and T. Sugie : Cooperative control for target-capturing task based on a cyclic pursuit strategy, Automatica, Vol. 43, Issue 8, pp. 1426-1431, 2007.

[7] Y. Kobayashi, K. Otsubo, and S. Hosoe : Design of Decentralized Capturing Behavior by Multiple Mobile Robots, in Proc. of 2006 IEEE Workshop on Distributed Intelligent Systems, pp.13-18, 2006.

[8] T. H. Chung, V. Gupta, J. W. Burdick and R. M. Murray : On a Decentralized Active Sensing Strategy using Mobile Sensor Platforms in a Network, in Proc. of the IEEE Conference on Decision and Control, 2004.

[9] D. J. Klein and K. A. Morgansen : Controlled Collective Motion for Trajectory Tracking, in proc. of the 2006 American Control Conference, pp. 5269-5275, 2006.

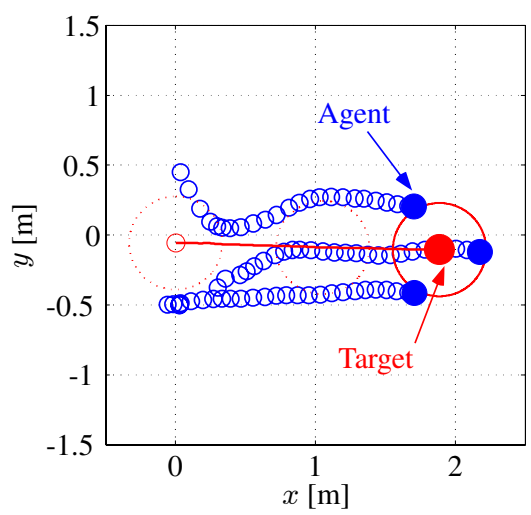

Fig. 7. Case 2 : Trajectories of agents and target-object

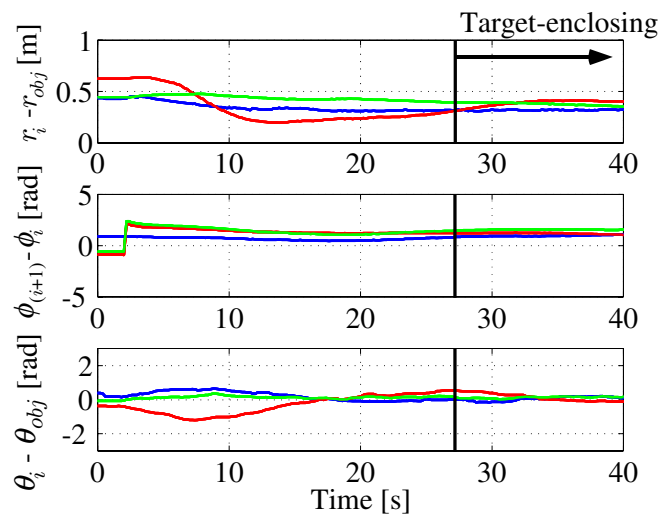

Fig. 8. Case 2 : Time plots of $r_{i}-r_{o b j}, \phi_{(i+1)}-\phi_{i}$ and $\theta_{i}-\theta_{o b j}$

[10] H. Yamaguchi : A distributed motion coordination strategy for multiple nonholonomic mobile robots in cooperative hunting operations, Robotics and Autonomous Systems, Vol. 43, Issue 4, pp. 257-282, 2003.

[11] D. J. Klein, C. Matlack and K. A. Morgansen : Cooperative Target Tracking using Oscillator Models in Three Dimensions, in proc. of the 2007 American Control Conference, pp. 2569-2575, 2007.

[12] J. A. Marshall, M. E. Broucke and B. A. Francis : Formations of Vehicles in Cyclic Pursuit, IEEE Trans. Automatic Control, Vol. 49, no. 11, pp. 1963-1974, 2004

[13] J. A. Marshall, M. E. Broucke and B. A. Francis : Pursuit formations of unicycles, Automatica, Vol. 42, Issue 1, pp. 3-12, 2006.

[14] W. Ren : Consensus Based Formation Control Strategies for Multivehicle Systems, in proc. of the 2006 American Control Conference, pp. 4237-4242, 2006.

[15] W. Ren : Consensus Seeking in Multi-vehicle Systems with a Timevarying Reference State, in proc. of the 2007 American Control Conference, pp. 717-722, 2007.

[16] T. Namerikawa and C. Yoshioka : Formation Control of Nonholonomic Multi-Vehicle Systems via Virtual Structure, in Proc. of the Fourth International Conference on Computational Intelligence Robotics and Autonomous Systems, pp. 202-207, 2007.

[17] C. Yoshioka and T. Namerikawa : Observer-based Consensus Control Strategy for Multi-Agent System with Communication Time Delay, in Proc. of IEEE Multi-conference on Systems and Control, 2008.

[18] W. Dong and J. A. Farrell : Decentralized Cooperative Control of Multiple Nonholonomic Systems, in proc. of the IEEE Conference on Decision and Control, 2007.

[19] Z. Lin, B. Francis and M. Maggiore : Necessary and sufficient graphical conditions for formation control of unicycles, IEEE Trans. on Automatic Control, Vol. 50, No 1, pp. 121-127, 2005.

[20] C. Godsil and G. Royle, Algebraic Graph Theory, Springer, 2001. 